\title{
Nuevo Código de Ética Profesional Odontológica en Chile: Contribución para una Relación Médico-Paciente Moderna
}

\author{
New Code of Professional Ethics for Dentist in Chile: \\ Contribution for a Modern Doctor-Patient Relationship
}

Lidia Bravo R. ${ }^{1} \&$ Denisse Lagos Tissie ${ }^{1,2}$

BRAVO, R. L. \& LAGOS, T. D. Nuevo código de ética profesional odontológica en Chile: Contribución para una relación médico-paciente moderna. Int. J. Odontostomat., 11(4):419-424, 2017.

RESUMEN: El objetivo de este estudio es analizar el Código de Ética propuesto recientemente por el Colegio de Cirujano Dentistas de Chile a la luz de la revisión de los códigos vigentes en otros países del mundo, discutiendo los temas que son más frecuentemente regulados en ellos, en lo referente a la relación médico-paciente. Se llevó a cabo una búsqueda en páginas web de acceso público de los Códigos de Ética Odontológica de Chile, Unión Europea, Estados Unidos, Australia y algunos países latinoamericanos. Se realizó la dentificación, clasificación, selección y análisis de los temas considerados más relevantes. En lo referido a la relación odontólogo-paciente, los temas seleccionados fueron: consentimiento informado, confidencialidad y secreto profesional, discriminación en la atención, atenciones de urgencia, aplicación de estándares de calidad actualizados, y especialización. Existen grandes diferencias en cuanto a la profundidad al tratar cada uno de los temas y en el alcance de las normas dictadas. Creemos que estas diferencias podrían deberse, por un lado, a la diversidad sociocultural de los países de la muestra analizada y, por otro, a las distintas legislaciones existentes sobre estos temas en cada uno de los países de la muestra.

PALABRAS CLAVE: códigos de ética profesional, relación médico-paciente, consentimiento informado.

\section{INTRODUCCIÓN}

Desde hace décadas, los avances de la medicina, el desarrollo de nuevas técnicas de diagnóstico y tratamiento, y los cambios que ha sufrido la relación médico-paciente (RMP), han elevado la complejidad del acto médico de manera creciente. Las desavenencias inevitables que surgen en la relación clínica, sobrepasan muchas veces el alcance de la ley, siendo necesario aplicar principios éticos en su resolución. Estos principios, en el caso de las profesiones de la salud, se encuentran contenidos en los respectivos Códigos de Ética Profesional.

La finalidad de un Código de Ética Profesional es servir como normas de conducta para los miembros asociados al colegio profesional del cual emana. En el caso de los Códigos profesionales del área de la salud, éste contiene las normas éticas que deben seguir los miembros en sus relaciones profesionales con sus pacientes, colegas, otros profesionales médicos y personal auxiliar, con los miembros del colegio al que pertenecen y los de otras organizaciones $y$, finalmente, con la comunidad y la sociedad en su conjunto. En la RMP, una relación de confianza y una comunicación fluida entre las partes es fundamental para la prevención de la judicialización de los inevitables conflictos que se suscitan en las relaciones humanas.

El año 2016 el Colegio de Cirujano Dentistas de Chile dio a conocer el documento que dará cuerpo al nuevo Código de Ética de la profesión, que reemplazará las actuales Normas de Conducta Ética, vigentes desde 1985.

\footnotetext{
${ }_{1}^{1}$ Programa de Formación de Especialistas en Odontología Legal y Forense, Facultad de Odontología, Universidad de Chile. Santiago, Chile. ${ }^{2}$ Medicina Legal Odontológica, Facultad de Odontología, Universidad de Chile. Santiago, Chile.
} 
El propósito del presente estudio es analizar este Código a la luz de la revisión de los códigos vigentes en otros países del mundo, discutiendo los temas que son más frecuentemente regulados en ellos, en lo referente a la RMP.

\section{MATERIAL Y MÉTODO}

Con fecha octubre del año 2016, se realizó una búsqueda en las páginas web de algunas de las principales Asociaciones Profesionales Odontológicas del mundo, desde donde se obtuvo los Códigos de Ética que los rigen. Se analizó el contenido de los Códigos del Council of European Dentist, del Dental Board of Australia, de la American Dental Association, de la Asociación Dental Mexicana, del Colegio de Odontólogos de la Provincia de Buenos Aires, del Consejo Federal de Odontología de Brasil, del Colegio Odontológico del Perú y del Colegio de Cirujano Dentistas de Chile.

Para facilitar su análisis, se efectuó la clasificación de los diferentes artículos de estos códigos, según el tema del que trataban, en dos grandes grupos: «relación del odontólogo con sus pacientes» y «relación con los colegas y la sociedad en su conjunto». Para el presente estudio, se seleccionaron los temas relativos a la RMP: consentimiento informado, confidencialidad y secreto profesional, discriminación en la atención, atenciones de urgencia, aplicación de estándares de calidad actualizados y especialización.

\section{RESULTADOS}

\section{Códigos analizados}

1. Council of European Dentist (2007): 28 artículos, divididos en 4 áreas: Compromiso con el paciente, Compromiso con el público, Práctica profesional y Comercio electrónico.

2. Dental Board of Australia (2014): 61 artículos, divididos en 11 áreas: Introducción, Proporcionar buen cuidado, Trabajo con pacientes o clientes, Trabajo con otros profesionales, Trabajo en el sistema de salud, Minimizar riesgos, Mantener rendimiento profesional, Comportamiento profesional, Garantizar la salud del profesional, Enseñanza, supervisión y evaluación e Investigación.
3. American Dental Association (2016): 29 artículos, divididos en 5 áreas: Autonomía, No maleficencia, Beneficencia, Justicia y Veracidad.

4. Asociación Dental Mexicana (1996): 54 artículos, sin subdivisiones.

5. Colegio de Odontólogos de la Provincia de Buenos Aires (2003): 28 artículos, divididos en 7 áreas: De la conducta personal y profesional en general, De los pacientes en particular, De las relaciones con los colegas y otros profesionales, Con relación a los mecánicos dentales, Del secreto profesional, De la transgresión a las normas del código, Del odontólogo auditor.

6. Consejo Federal de Odontología de Brasil (2013): 60 artículos, divididos en 19 áreas: Disposiciones Generales, Derechos fundamentales, Deberes fundamentales, Auditorías y pericias odontológicas, Relaciones con los pacientes y con el equipo de salud, Secreto profesional, Documentos odontológicos, Honorarios profesionales, Especialidades odontológicas, Odontología hospitalaria, Entidades con actividades en el ámbito odontológico, Responsables técnicos y propietarios inscritos, Docencia, Donación, trasplante, banco de órganos, tejidos y biomateriales, Entidades gremiales, Anuncios, propaganda y publicidad, Investigación científica, Penas y sus aplicaciones y Disposiciones finales.

7. Colegio Odontológico del Perú (2016): 96 artículos, divididos en 2 áreas: Parte general y Parte especial. Esta última, dividida en 9 capítulos: Del ejercicio profesional, De la expedición de certificados, odontograma e historia clínica, De las relaciones profesionales, Del secreto profesional, Del funcionario público, Del magisterio, De la investigación y publicaciones científicas, De la promoción y publicidad de la actividad profesional, De los honorarios profesionales.

8. Colegio de Cirujano Dentista de Chile (2016): 58 artículos, divididos en 6 áreas: Disposiciones generales, De la publicidad, Relación del cirujano dentista y su ejercicio profesional, De la relación del cirujano dentista con su paciente, familiares y representantes, Relaciones personales, interprofesionales y personal de soporte y Relaciones del cirujano dentista con su Colegio.

\section{Análisis y comparación}

Dentro del grupo "relación del odontólogo con sus pacientes", los 6 temas más relevantes que se analizaron son los siguientes (Tabla I): 
Tabla I. Comparación de temas relevantes sobre RMP tratados en los Códigos de Ética analizados.

\begin{tabular}{|c|c|c|c|c|c|c|c|c|}
\hline Relación Odontólogo-Paciente & Chile & UE & Austr & EEUU & Méx & Bs A & Brasil & Penú \\
\hline \multicolumn{9}{|l|}{ Consentimien to informado } \\
\hline Debe realizarse antes de iniciar cualquier tratamiento & si & si & si & si & si & si & si & si \\
\hline Debe ser realizado directamente por el dentista tratante & si & si & si & si & si & no & no & si \\
\hline Debe realizarse siempre por escrito & si & no & no & no & si & no & no & si \\
\hline Debe realizarse por escrito cuando existe riesgo grave & - & no & si & no & - & no & no & - \\
\hline Se realiza con tutor en caso de pacientes no aptos & si & si & si & no & no & no & si & si \\
\hline Involucrar al niño o joven en la toma de decisiones & no & no & si & no & no & no & no & no \\
\hline Informar al paciente fallos o complicaciones y discutir soluciones & si & si & si & no & no & si & no & no \\
\hline Confidencialidad y Secreto profesional & Chile & UE & Austr & EEUU & Méx & Bs A & Brasil & Pení \\
\hline El Dentista debe resguardar información del paciente & si & si & si & $\mathrm{si}$ & si & si & si & si \\
\hline Asegurar que colaboradores también respeten confidencialidad & si & si & si & no & no & no & si & si \\
\hline Entregar copia ficha clínica cuando el titular, tutor, justicia lo requiera & no & si & si & si & si & si & si & si \\
\hline Excepciones: enf. de notificación obligatoria y seguros de salud & no & no & si & no & si & si & si & si \\
\hline Uso de datos en investigación sólo con la autoriza ción del paciente & no & no & si & no & no & no & si & si \\
\hline Uso de datos en docencia sólo con la autorización del paciente & no & no & no & no & no & no & si & si \\
\hline
\end{tabular}

\section{No Dis criminar}

A ningún individuo o grupo de individuos

Por raza, credo, color, sexo, nacionalidad, enfermedad infecciosa Por raza, credo, color, sexo, nacionalidad, enfermedad, edad, rango social Derecho a se leccionar pacientes por razones personales salvo en urgencias Derecho a seleccionar según habilidades

Derecho a derivar por conflictos morales o religiosos

Derecho a derivar ante discrepancias en los criterios clínicos

Derecho a derivar ante incumplimiento del paciente

Derecho a derivar cuando se quiebre la RMP

Obligación de realizar atenciones de urgencias

A pacientes propios y en general

$\mathrm{Y}$ referir luego al tratante original

\section{Estándares de calidad}

Aplicar odontología basada en evidencia

Perfeccionamiento permanente del dentista

Usar instalaciones, fármacos y materiales probados

Mantener auditorias, respetando autonomía profesional

$\begin{array}{llllllll}\text { Chile } & \text { UE } & \text { Austr } & \text { EEUU } & \text { Méx } & \text { Bs A } & \text { Brasil } & \text { Perú } \\ \text { si } & \text { si } & \text { si } & - & - & - & \text { si } & - \\ - & - & - & \text { si } & \text { si } & - & - & - \\ - & - & - & - & - & \text { si } & - & \text { si } \\ - & - & - & \text { si } & \text { si } & \text { si } & - & - \\ - & - & \text { si } & - & - & - & - & - \\ - & \text { si } & \text { si } & - & - & - & - & - \\ - & - & - & - & - & - & \text { si } & \text { si } \\ - & - & - & - & - & - & - & \text { si } \\ \text { si } & - & - & - & - & - & \text { si } & - \\ & & & & & & & \\ \text { Chile } & \text { UE } & \text { Austr } & \text { EEUU } & \text { Méx } & \text { Bs A } & \text { Brasil } & \text { Perú } \\ \text { no } & \text { no } & \text { si } & \text { si } & \text { si } & \text { si } & \text { si } & \text { si } \\ - & - & \text { no } & \text { si } & \text { si } & \text { no } & \text { no } & \text { no }\end{array}$

Chile UE Austr EeUU Méx Bs A Brasil Perú

si si si no si si si si

si si si $\quad$ si $\quad$ si $\quad$ no $\quad$ si $\quad$ si

no no si si si si si si

no no no no no no si no

\section{Especialidades}

Ejercer sólo la especialidad obtenida en institución acre ditada Al anunciar especialidad, se debe limitar la practica a esa área
Chile UE Austr EeUU Méx Bs A Brasil Perú

si no no si si no si si

Chile:Colegio de Cirujano Dentista de Chile. UE: Council of European Dentist. Austr: Dental Board of Australia. EEUU: American Dental Association. Méx: Asociación Dental Mexicana. Bs A: Colegio de Odontólogos de la Provincia de Buenos Aires. Brasil: Consejo Federal de Odontología de Brasil. Perú: Colegio Odontológico del Perú.

1. Consentimiento Informado (CI): "proceso mediante el cual a un paciente se le proporciona la información suficiente para tomar una decisión razonada acerca del procedimiento propuesto. Es otorgado por el paciente sin ninguna coacción o fraude, basado en su entendimiento razonable de lo que sucederá, incluyendo la necesidad de realizar el tratamiento, los beneficios y riesgos de éste, la existencia de cualquier procedimiento alternativo disponible y los riesgos de la no aceptación del tratamiento" (García, 2006).
Todos los códigos revisados concuerdan en que este proceso debe ser llevado a cabo siempre. Todos, excepto el argentino y el brasileño, señalan explícitamente que debe ser realizado por el dentista tratante. Sólo los códigos chileno, mexicano y peruano señalan que debe firmarse un documento que certifique la realización del $\mathrm{Cl}$. Los códigos de Chile, Unión Europea (UE), Australia, Brasil y Perú señalan la necesidad de que un tutor participe del $\mathrm{Cl}$ en los casos en que el paciente no sea apto para darlo. 
2. Confidencialidad y Secreto Profesional: la confidencialidad en la atención médica es el derecho que tiene un paciente de que su información y datos sensibles no sean divulgados por el personal que lo atiende. El secreto médico es la obligación que tienen los médicos, odontólogos y todo el personal de salud, de guardar reserva de los hechos conocidos por ellos durante el ejercicio laboral.

En todos los Códigos estudiados se estipula el derecho a confidencialidad de los pacientes. En todos, excepto el chileno, se consigna el derecho del paciente, su representante o heredero, a recibir una copia de su ficha clínica cuando se solicite o cuando la ley lo requiera. Los Códigos de Australia, México, Argentina, Brasil y Perú hacen referencia a la obligación de romper el secreto profesional en algunas circunstancias específicas. Los Códigos de Australia, Brasil y Perú prohíben explícitamente el uso de los datos del paciente en investigación sin su consentimiento.

3. Discriminación: según Gracia, la Justicia es uno de los principios de la bioética que constituye, junto con la No Maleficencia, la "Ética de Mínimos". Esto implica que la aplicación del principio de Justicia es independiente de la voluntad del paciente y debe ser garantizado por el Estado. El Principio de Justicia en medicina nos dice que todos deben ser tratados sin ningún tipo de discriminación (Gracia, 1991).

Todos los códigos incluidos en la presente revisión relevan la importancia de no discriminar arbitrariamente al seleccionar pacientes, sin embargo, la mayoría consigna, por otro lado, el derecho del dentista de seleccionar a sus pacientes según otros criterios o de derivarlos en determinadas circunstancias.

4. Atenciones de urgencia: son aquellas que tratan patologías de aparición súbita, que generalmente provocan dolor y demandan atención inmediata e impostergable.

En todos los códigos, excepto en el de Chile y la UE, se consigna la obligación de realizar atenciones de urgencias al público en general. En los de Estados Unidos (EEUU) y México se señala, además, la obligatoriedad de referirlos luego a sus respectivos tratantes.

5. Aplicación de estándares de calidad: en la década de los noventa, nace el concepto de Medicina Basada en la Evidencia, definida como "el uso explícito, racional y juicioso de la mejor evidencia disponible en la toma de decisiones clínicas, incorporando los valores y las preferencias de los pacientes" (Sackett et al., 1996). En nuestra área, la Odontología Basada en Evidencia (OBE) es la norma que se aplica en la creación de los protocolos de atención odontológica.

En seis de los Códigos revisados (Chile, UE, Australia, México, Argentina y Brasil), se consigna que es obligación del dentista tratante aplicar OBE, proporcionando tratamientos debidamente probados. En todos los códigos, salvo Chile y UE, se señala que sólo se deben utilizar instalaciones, materiales y fármacos aceptados y seguros.

6. Especialización: Los Códigos de Chile, EEUU, México, Brasil y Perú señalan que sólo se puede publicitar y ejercer la especialidad obtenida en alguna institución acreditada. La UE hace hincapié en que se debe cumplir la legislación respecto a la práctica profesional.

\section{DISCUSIÓN}

El análisis de estos diferentes Códigos de Ética Profesional permite tener un panorama amplio sobre la problemática ética a la que nos enfrentamos como profesión, a nivel mundial. Todos los Códigos analizados coinciden en cuáles son las principales temáticas éticas y deontológicas que interesan a la profesión. Sin embargo, existen diferencias en cuanto a la profundidad al tratar cada uno de estos temas y en el alcance de las normas dictadas. Creemos que estas diferencias podrían deberse, por una parte, a la diversidad sociocultural de los países de la muestra analizada y por otra, estar relacionado con las distintas legislaciones existentes sobre estos temas en cada uno de los países de la muestra. En Chile existe normativa legal que regula la mayoría de los temas analizados en este artículo, como se presenta a continuación.

Consentimiento informado. El cambio desde el modelo paternalista en medicina a la instalación del concepto de autonomía se ve materializado en este proceso. Llama la atención que en tres países latinoamericanos, entre ellos Chile, el Código exija la firma de un documento en cada atención, restándole de alguna manera valor al consentimiento verbal. De hecho, en nuestro país, además, la Ley 20.584 ordena la firma del $\mathrm{Cl}$ en cada atención invasiva. Dado que la mayoría de las prestaciones odontológicas son invasivas y contamos con escaso tiempo para la aten- 
ción, especialmente en el sistema público de salud, la firma de un documento de $\mathrm{Cl}$ es impracticable para cada atención. Podría incluso volverse desaconsejable también, ya que puede llegar a instalar la desconfianza en una RMP ya establecida.

Por otro lado, ni el Código ni la Ley chilena hacen referencia al proceso de Asentimiento Informado en el caso de aquellos pacientes que no pueden otorgar su consentimiento, como ocurre con los niños o pacientes en situación de discapacidad intelectual. El Cirujano Dentista, al enfrentarse a estos pacientes, no cuenta con una norma ética que lo ayude en la toma de decisiones clínicas durante la atención.

Confidencialidad y Secreto Profesional. El secreto profesional es otro tema ampliamente tratado en los Códigos estudiados. Esta obligación se funda también en normas legales, que en Chile podemos encontrar en el Código Penal (Art.247), Código Procesal Penal (Art.303), Ley 20.584 (Art.5c, 12 y 13), Ley 18.834 y Ley 19.628.

Si bien todos concuerdan en que es un deber inquebrantable del cirujano dentista para con sus pacientes, la utilización de los datos clínicos e imágenes como material docente no está tratada debidamente en la mayoría de ellos, a pesar de ser un recurso ampliamente usado. La privacidad de los pacientes no debe verse supeditada a la utilidad de los datos como medio de aprendizaje.

Discriminación: El derecho a la salud es un derecho humano fundamental. El derecho a ser atendido en el ámbito médico sin que medie discriminación arbitraria alguna, está garantizado en la Ley 20.584 (Art.2), en la Constitución Política de la República (Art.19) y en los Tratados Internacionales ratificados por el Estado. Sin embargo, sugerimos que el Código chileno se pronuncie respecto a la selección de paciente según las habilidades o preferencias del tratante (salvo en situaciones de emergencia) lo cual ya ha sido incluido en otros Códigos del mundo, y que habitualmente se realiza en la práctica clínica.

Atención de Urgencia: El Código de Ética chileno no consigna ninguna obligación ética específica en la realización de atenciones de urgencia. Sin embargo, en Chile, bajo el Decreto Supremo No 44/07, las Garantías Explícitas en Salud incluyen la atención de Urgencias Odontológicas Ambulatoria. De esta manera, se garantiza atención inmediata para la resolución de éstas.
Aplicación de estándares de calidad: Siete de los Códigos analizados explicitan la obligación de la aplicación de la OBE en el tratamiento clínico cotidiano. En cambio, el de EEUU pone hincapié sólo en la actualización permanente de conocimientos y habilidades. En Chile, el derecho a ser atendido según las mejores normas vigentes se garantiza, además, en la Ley 20.584 (Art.4). Sin embargo, poco se dice sobre los estándares de calidad de los procedimientos diagnósticos y el uso, a veces indiscriminado, de exámenes radiográficos para tal propósito.

Especialización: En la mayoría de los Códigos, incluyendo el chileno, se ordena ejercer sólo las especialidades que se hayan obtenido en instituciones acreditadas. Sin embargo, en Chile no existe ley de especialidades odontológicas que reglamente el campo de acción clínica del cirujano dentista general, lo que genera un vacío en el ámbito de la calidad de las atenciones odontológicas que se entrega a los pacientes. La normativa al respecto sólo hace referencia a la Acreditación de Especialidades y Subespecialidades Médicas y Odontológicas, en la Ley 19.937 y el Decreto Supremo No 57/07 y su modificación Decreto Supremo No 114/10.

La modernización continua de los Códigos de Ética Odontológicos es una necesidad que nace del acelerado avance técnico-científico y de la evolución de las relaciones interpersonales en el ámbito médico, junto con un cambio en las formas de interacción social. El uso de internet permite a los pacientes no sólo el acceso a contenidos sobre diversas condiciones de salud y tratamientos (no siempre verídicos o exactos), sino que también un acercamiento a la vida personal del dentista tratante que puede llegar a entorpecer la RMP. Muchos Códigos de Ética empiezan a dar cuenta de esta realidad, orientando a un uso criterioso de las redes sociales virtuales. Sería un aporte valioso tratar este tema en el Código chileno, junto con otros como el asentimiento informado, el derecho a la selección de pacientes según preferencias clínicas y el uso de información confidencial como material docente.

En aquellos aspectos de nuestro ejercicio sobre los que no existen normas legales, el autocontrol ético profesional juega un rol preponderante: "saber ser", ser prudente en nuestras decisiones y procurar hacerle el bien a nuestros pacientes nos vuelve profesionales íntegros y propicia la cercanía en la RMP. 
BRAVO, R. L. \& LAGOS, T. D. New code of professional ethics for dentist in Chile: Contribution for a modern doctorpatient relationship. Int. J. Odontostomat., 11(4):419-424, 2017.

ABSTRACT: The objective of this study was to analyze the new Code of Ethics recently presented by the Chilean Colegio de Cirujano Dentistas, in light of the results obtained in the revision of the Codes of other countries, focusing on the doctor-patient relationship. Search and obtain from public websites, the Codes of Ethics from the European Union, the United States, Australia and some Latin American countries. Identification, classification, selection and analysis of the most relevant topics. Regarding the dentist-patient relationship, the selected topics were: Informed consent, confidentiality and professional secrecy, discrimination in care, emergency care, application of up-to-date quality standards and specialization. We observed important differences in regards to the depth in which each topic is covered, and the scope of the established norms. We believe that these differences may arise as a result, on the one hand, of the sociocultural diversity of the analyzed countries and, on the other hand, of the different legislations that these countries may have in place on those topics.

KEY WORDS: professional ethics codes, doctorpatient relationship, informed consent.

\section{REFERENCIAS BIBLIOGRÁFICAS}

Asociación Dental Mexicana. Código de Ética, 1996. http:// admmexico.org/codigo-de-etica-adm/.

Código Penal. Diario Oficial de la República de Chile. Santiago, Chile, 12 de noviembre de 1874.

Código Procesal Penal. Diario Oficial de la República de Chile. Santiago, Chile, 12 de octubre de 2000.

Colegio de Cirujano Dentista de Chile, Código de Ética, 2016.

Colegio de Odontólogos de la Provincia de Buenos Aires Consejo Superior. Código de Ética, 2003. http:// www.cosucoba.org.ar/

Colegio Odontológico del Perú. Código de Ética y Deontología, 2016. http://www.cop.org.pe/legislacion.

Consejo Dental de Australia. Código de Conducta para Profesionales de la Salud, 2014. http://www. dentalboard.gov.au/Codes-Guidelines/Policies-CodesGuidelines.aspx

Consejo Federal de Odontología de Brasil, Código de Ética Odontológico, 2012. http://cfo.org.br/legislacao/codigos/

Decreto con Fuerza de Ley $N^{\circ} 29$, que fija el texto refundido, coordinado y sistematizado de la Ley № 18.834 , sobre Estatuto Administrativo. Diario Oficial de la República de Chile, Santiago, Chile, 16 de marzo de 2015.

Decreto $\mathrm{N}^{\circ} 44$ de la Superintendencia de Salud. Aprueba garantías explícitas en salud del régimen general de garantías en salud. Diario Oficial de la República de Chile, Santiago, Chile, 31 de enero de 2007.
Decreto Supremo No 114/10 de los Ministerios de Salud y Educación, que modifica el Decreto № 57, de 2007. Diario Oficial de la República de Chile, Santiago, Chile, 17 de febrero de 2011.

Decreto Supremo N 57/07 del Ministerio de Salud, Reglamento de certificación de las Especialidades y Subespecialidades de los Prestadores Individuales de Salud y de las Entidades que la otorgan. Diario Oficial de la República de Chile, Santiago, Chile, 6 de noviembre de 2008.

García, F. El odontólogo frente al consentimiento informado ¿qué hacer? En: Cardozo, C; Rodríguez, E; Lolas, F; Quezada, A Editores, Ética y Odontología. Una Introducción. Santiago, Chile: Editorial Universidad de Chile (CIEB), 2006. pp. 105-16,

Gracia, D. Procedimientos de Decisión en Ética Clínica. Madrid, España: Editorial Eudema S. A., 1991. pp.12434.

Ley 19.628, sobre Protección de la Vida Privada. Diario Oficial de la República de Chile, Santiago, Chile, 28 de agosto de 1999.

Ley 19.937 , que modifica el D.L. N ${ }^{\circ} 2.763 / 79$, con la finalidad de establecer una nueva concepción de la autoridad sanitaria, distintas modalidades de gestión y fortalecer la participación ciudadana. Diario Oficial de la República de Chile, Santiago, Chile, 24 de febrero de 2004.

Ley 20.584 sobre Derechos y Deberes de los Pacientes. Diario Oficial de la República de Chile, Santiago, Chile, 24 de abril de 2012.

Principio de Ética y Código de Conducta Profesional de la Asociación Dental Americana (2016). http://www.ada.org/ en/about-the-ada/principles-of-ethics-code-ofprofessional-conduct.

Sackett, D. L.; Rosenberg, W. M.; Gray, J. A.; Haynes, R. B. \& Richardson, W. S. Evidence based medicine: what it is and what it isn't. BMJ, 312:71-2, 1996.

Unión Europea del Consejo Europeo de Dentistas. Código de Ética para Dentistas, 2007. http://www.cedentists.eu/ ced-code-of-ethics.html.

Dirección para correspondencia

Denisse Lagos Tissie

Programa de Formación de Especialistas en

Odontología Legal y Forense

Facultad de Odontología

Universidad de Chile

Santiago - CHILE

Email: dlagos@odontologia.uchile.cl

Recibido : 24-07-2017

Aceptado: 14-11-2017 